\title{
Erratum to: Efficacy of two different surgical techniques combined in the treatment of rectocele
}

\author{
Fabio Gaj · Antonello Trecca • Jacopo Andreuccetti •
}

Pietro Crispino

Published online: 20 July 2012

(C) Springer-Verlag 2012

Erratum to: Updates Surg (2012) 64:107-112

DOI 10.1007/s13304-012-0149-0

Unfortunately, one of the authors name has been misspelled in the original publication. The correct spelling should be Jacopo Andreuccetti.

The online version of the original article can be found under doi:10.1007/s13304-012-0149-0.

F. Gaj · J. Andreuccetti

Dipartimento di Chirurgia Generale e Trapianti d'Organo,

Istituto "Paride Stefanini”, Università 'La Sapienza' di Roma,

Via Reno 30, 00198 Rome, Italy

e-mail: fagaj@tin.it

A. Trecca · J. Andreuccetti

Unità di Endoscopia e Gastroenterologia Operativa "Fabio Di

Giovambattista" UEGO FdG Roma, Rome, Italy

J. Andreuccetti · P. Crispino $(\bowtie)$

UOC Medicina, D’Urgenza, accettazione PSA, Ospedale San

Giovanni, ASP Potenza, PO di Lagonegro (PZ), Italy

e-mail: picrispino@libero.it 